\title{
Two Types of Renal Mechanoreceptors
}

\author{
Yasumi Uamida, M.D., Kazuaki Kamisaka, M.D., \\ and Hideo Ueda, M.D.
}

\section{SUMmary}

Two types of the afferent impulses were recorded in the renal nerves of the mongrel dogs; the ones of low voltage, spontaneously discharging and of slowly adapting units (type A), and the others of higher voltage and threshold, not spontaneously discharging and of rapidly adapting units (type B). Type A impulses consisted of the ones of irregularly discharging and those of regularly discharging at each ascent of intrarenal pressure waves. Type A impulses increased or decreased concomitantly with intrarenal pressure on changing the pressure to different directions from either renal arterial or venous pressures. On the other hand, type B impulses appeared transiently at the fall or rise in the pressure.

The discharges of type $\mathbf{B}$, elicited by elevating intrarenal pressure perfusing Ringer's solution into the pelvis, to abnormally high levels, disappeared on reducing intrarenal pressure by occluding the renal artery whilst elevating venous pressure.

In conclusions, the renal mechanoreceptors of both types were excited by mechanically induced alterations in intrarenal pressure.

\section{Additional Indexing Words :}

Intrarenal pressure Renal arterial pressure Renal venous pressure Afferent impulses

$\mathrm{T}$

HE nerve endings connecting to the myelinated afferent fibers are observed in the kidney. ${ }^{2,43,6), 8)}$ Some of them have an action inhibiting the efferent sympathetic discharge and producing a fall in the systemic arterial pressure. ${ }^{1,12)}$ The afferent impulses from these nerve endings are evoked or increased by mechanical elevation of either the renal arterial or venous pressure. ${ }^{3,9,11)}$ It was observed in our previous experiments that the responses to mechanical stimuli differed by the fibers and that a rise in the arterial pressure evoked the impulses indirectly through the increased intrarenal pressure. ${ }^{12}$

The present experiments were undertaken to classify the impulses of the nerve endings from their response patterns to the mechanically induced alterations in the renal hemodynamics and to elucidate, by changing the venous pressure to the different direction with either renal arterial or intrarenal pressure, whether the renal venous pressure stimulates the nerve endings directly. Tokyo.

From the Second Department of Internal Medicine, Faculty of Medicine, University of Tokyo,

Received for publication October 13, 1970. 


\section{Materials and Methods}

Sixteen mongrel dogs were used for the present studies. The dogs were anesthetized with pentobarbital sodium (35-40 mg./Kg.) injected intravenously. The respiration was maintained with an endotracheal tube attached to a respirator pump. They were immobilized on their right sides. The left flank was opened and the retroperitoneum was resected to pull out the left kidney. The surrounding connective tissues of the renal nerves were resected. The renal nerve was divided into thin filaments, the cut central end was lifted up onto the small bipolar electrodes and was covered with warmed liquid paraffin. The afferent impulses were led off from these fibers, amplified for display on an oscilloscope, and were photographed. At the same time, the integral of number of the pulses was derived from the electroneurogram using a pulse-integrator, and was recorded on a pen-oscillograph simultaneously with the renal arterial, renal venous and intrarenal pressures. The pressures were recorded by Statham strain gauges connected to a needle inserted into the renal artery, to a needle inserted into the cortex or medulla, to a catheter introduced through the left spermatic vein into the renal vein, and to a catheter introduced into the pelvis. The threshold of the receptors (nerve endings) for the intrarenal pressure was studied in 5 dogs with elevating the pressure to various levels by occluding the renal vein or by perfusing Ringer's solution into the pelvis. The mode of adaptation of the receptors to elevated intrarenal pressure was examined in 3 dogs. Relations between the increase in number of the impulses and the rise in the intrarenal pressure were studied in 3 dogs with elevating the intrarenal pressure stepwise by occluding the renal vein or by perfusing Ringer's solution into the pelvis. In 3 dogs, the catheters from the left common carotid artery, right jugular vein and the left renal vein were connected with each other in $Y$ form. The blood from the common carotid artery was pumped out into the circuit by a Sigmamotor. Thus, the blood from both the renal vein and the artery flowed into the jugular vein. The screw calmps were attached both to the root of the renal artery and the jugular side of the circuit to change the resistance. A decrease in the intrarenal pressure was produced by lowering the renal arterial pressure while elevating the perfusion pressure of the renal vein. Thus, it became possible to observe the effects of the renal venous and intrarenal pressures separately.

\section{RESULTS}

Two types of the afferent impulses were recorded from the renal nerves in all 5 dogs examined; the ones of spontaneously discharging and the other of not spontaneously discharging. The latter appeared for the first time on compressing the kidney, occluding the renal vein or on elevating the intrapelvic pressure. The recordings of these two types of the impulses from the same preparation revealed that the former were of low voltage and the latter of higher voltage, presumably indicating that the former were from the small fibers and the latter, from large fibers (Fig. 1). Hereafter, the spontaneously discharging small impulses are called type $A$ impulses and the not spontaneously 


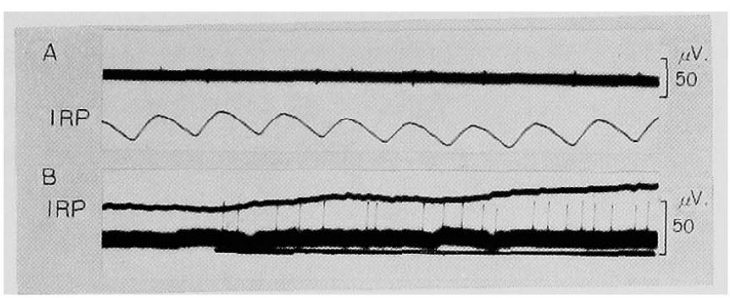

Fig. 1. Afferent impulses in the renal nerve.

A: irregularly and spontaneously discharging type A impulses.

B : type B impulses evoked by compressing the kidney at the horizontal bar.

The details are explained elsewhere.

IRP : intrarenal pressure.

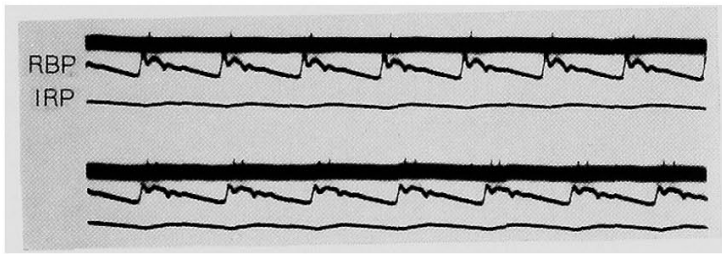

Fig. 2. Regularly discharging type $A$ impulses. One impulse for each ascent of the intrarenal pressure wave is observed in the upper recording, whereas 2 for each ascent in the lower recording in which the intrarenal pressure was elevated slightly.

RBP: renal arterial blood pressure.

discharging large impulses, type B impulses. Type A impulses consisted of two types of the impulses. The fibers of one type discharged irregularly apparently with little relation to the renal arterial, renal venous and to the intrarenal pressure waves, but increased or decreased with the artificially induccd changes in the intrarenal pressure. While, the fibers of another type discharged regularly at each ascent of the intrarenal pressure waves. In latter type, the number of the impulses per wave increased with a rise in the level of the intrarenal pressure, as shown in Fig. 2. The impulses of both type A and B units were led off separately, recorded simultaneously, and their thresholds were compared by elevating the intrarenal pressure to various levels. Type A impulses were recorded even when the pressure was near $10 \mathrm{~mm} . \mathrm{Hg}$, whereas type B impulses appeared for the first time at much higher levels, indicating that the threshold of the former for the intrarenal pressure was lower than that of the latter (Fig. 3). Type A impulses continued to increase for several tenths of seconds when the intrarenal pressure was elevated and maintained at a certain level almost constant. Type B impulses, on the contrary, appeared transiently and soon disappeared, indicating that the former adapted slowly and the latter rapidly to the increase in intrarenal pressure (Fig. 4). The impulses of both types increased with elevating the intrarenal pres- 


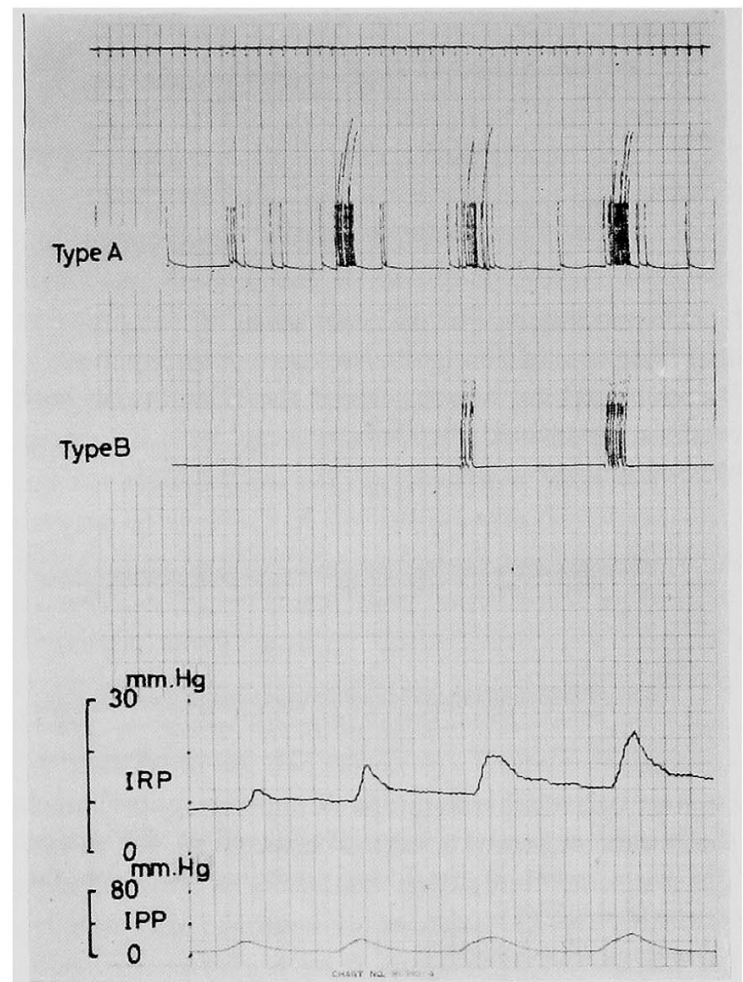

Fig. 3. Comparison of the thresholds of type A and B impulses. Ringer's solution was perfused into the pelvis to elevate the intrarenal pressure.

IPP: intrapelvic pressure.

At the top is shown the time scale.

Each segment indicates one second.

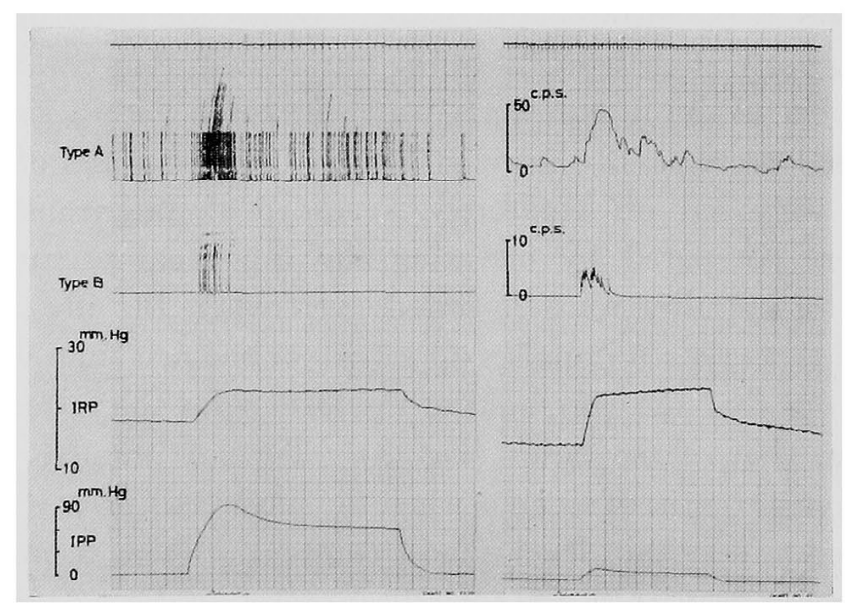

Fig. 4. Adaptation of type $\mathrm{A}$ and $\mathrm{B}$ units to an increase in the intrarenal pressure. Time constant of pulse integration in the right recording is longer than that in the left. 
sure by perfusion of Ringer's solution into the pelvis, but further increase was not observed over a certain level of the pressure (Fig. 5). Thus, the relationship between the increase in number of the impulses and the rise in the in-

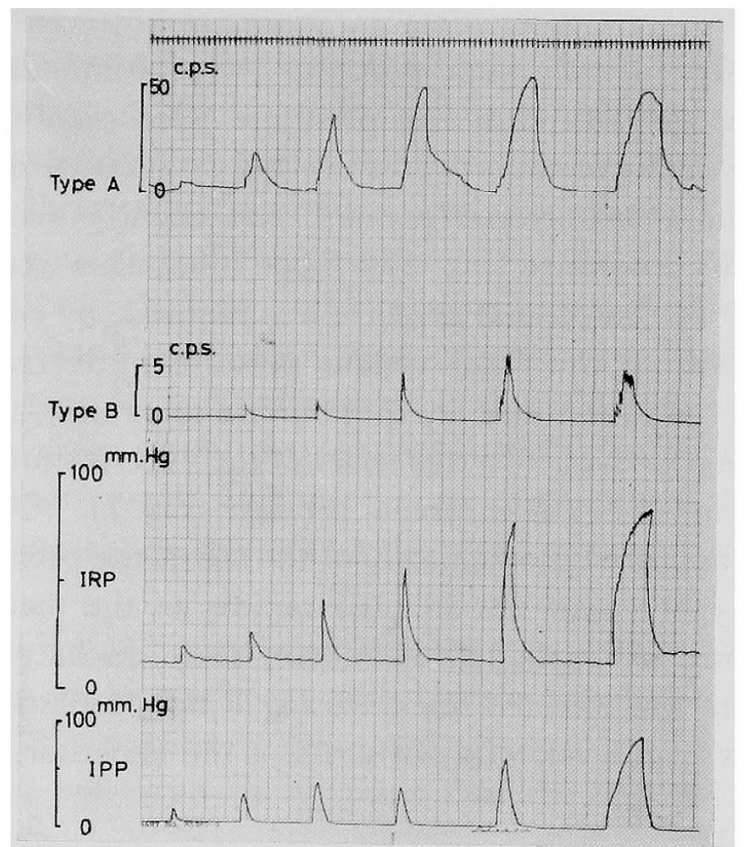

Fig. 5. Relations between the rise in the intrarenal pressure and the increase in impulses frequency.



Fig. 6. Left recording: effects of releasing the occlusion of the renal artery. Right recording: effects of rcnal vein occlusion. 
trarenal pressure in both types was represented by a linear pressure-characteristics curve. Type A impulses decreased with the fall in the intrarenal pressure produced by occluding the renal artery. After release of occlusion, they increased with the return of the pressure to the control level. In some experiments, however, a momentary increase was observed at the fall of the intrarenal pressure. By the same procedure, type B impulses appeared momentarily during the return of the pressure. Occlusion of the renal vein resulted in a rise in the intrarenal pressure and an increase in number of type A impulses. The renal arterial pressure was slightly decreased or almost unchanged by this procedure (Fig. 6). Type B impulses were also evoked by this procedure. The intrarenal pressure was reduced, by occluding the renal artery while elevating the renal venous pressure by increasing the outflow resistance of the perfusion circuit, to examine separately the effects on the receptors of these pressures. In this series of the experiments, type A impulses decreased with the fall in the intrarenal pressure (Fig. 7). On the other hand, type B impulses appeared momentarily at the fall of the pressure.

Although type B impulses adapted rapidly to the moderate rise in the intrarenal pressure as explained elsewhere, they discharged spontaneously when the pressure was elevated, by perfusing Ringer's solution into the pelvis to an abnormally high level. In this series of the experiments, the reduction

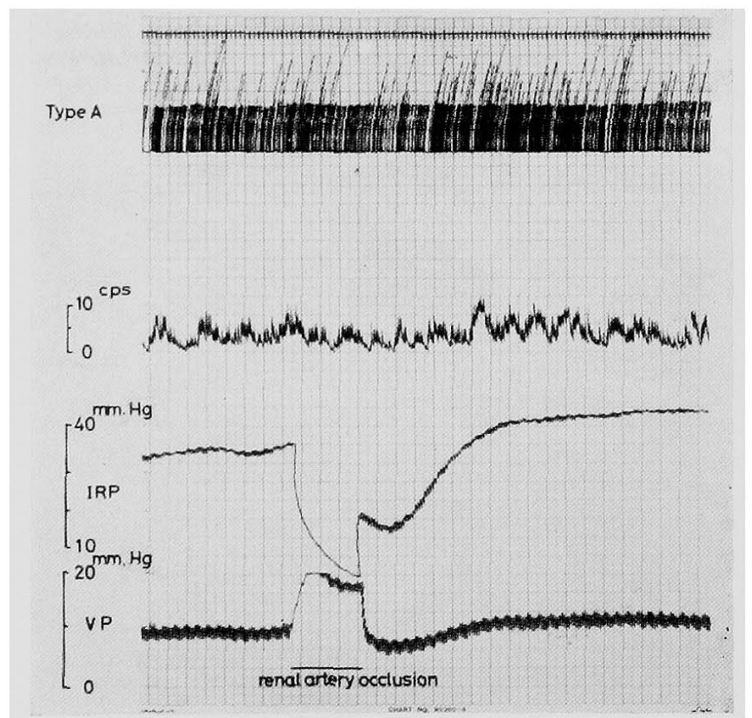

Fig. 7. The renal venous pressure was increased by elevating the perfusion pressure in the venous side of the circuit, while reducing the renal arterial pressure. By this procedure, a decrease both in the intrarenal pressure and impulse frequency were elicited. The upper and the middle tracings were derived from the same nerve preparation. Time constant for pulse integration in the middle is longer than that in the upper. 
in the intrarenal pressure resulted in the disappearance of the impulses.

\section{Discussion}

In our present experiments, the afferent impulses from the renal nerve were classified into two types mainly from their responses to the changes in the intrarenal pressure. To summarize, they were the ones of low voltage, spontaneously discharging and of slowly adapting units (type A) and the others of higher threshold and voltage, not spontaneously discharging and rapidly adapting units (type B). Asstrom and Crafoord recorded in rats both large and small spikes; the former of somewhat longer duration than the latter in response to the stimuli. However, they observed no spontaneous discharge. ${ }^{2}$ The lower spikes (type A) in the present experiments differ from those recorded by them, because those in our present experiments discharged spontaneously at normal intrarenal pressure. However, the type $A$ and $B$ impulses in our studies may respectively correspond to the small and large impulses recorded by them.

Type A impulses of regularly discharging units had a close relation to the intrarenal pressure waves, on the contrary, those of irregularly discharging units had apparently little relation to the renal arterial, renal venous nor to the intrarenal pressure waves. It is supposed that the regularly discharging receptors are more sensitive to the alterations in the intrarenal pressure or that they resided near the small renal arteries. It was not clarified what was the stimulus for the irregularly discharging impulses in usual conditions, although they were excited by the changes in the intrarenal pressure caused artificially. One possibility is that their differences in discharge patterns are due to the locations of the nerve endings.

The findings that type A fibers discharged spontaneously at normal intrarenal pressure support the possibility that they also discharge spontaneously in physiological conditions.

Type B fibers discharged in different ways from those of type A.

Although type B impulses might be from large fibers and those of type A from small fibers, it was not elucidated whether the differences in thrcsholds and adaptation are attributable to the characters of their nerve endings or the tissues in which they were imbedded.

A rise in the renal arterial pressure produced mechanically was accompained by an increase in the intrarenal pressure and also by an increase in number of the afferent impulses of both types. Occlusion of the renal vcin resulted in an increase in the intrarenal pressure and also in augmentation of the endings whereas resulted in a slight decrease or no change in the 
renal arterial pressure. Additionally, the impulses of both types changed to the same direction with the intrarenal pressure in the perfusion experiments in which the venous pressure was altered to different direction from the intrarenal pressure. These findings suggest that the stimulus for the receptors was not the renal arterial nor the renal venous pressure but the intrarenal pressure, and that both the arterial and venous pressure excited the receptors indirectly through the intrarenal pressure. In the present experiments, no impulses which had closer relations with either renal arterial or venous pressures were recorded, although the nerve endings connecting to the afferent fibers were observed histologically in the renal arterial and venous walls. ${ }^{3), 8 \text {; }}$ The afferent impulses recorded in the present experiments were, therefore, prcsumably from the receptors in the non-vascular spaces. In several experiments, a momental appearance of typc B impulses and an increasc of type A impulses were observed at the beginning of the fall in the intrarenal pressure produced by occluding the renal artery. These findings support the possibility that mechanically-induced alterations in the intrarenal pressure toward both increase and decrease suffice the stimuli.

In our previous studies, the impulses were recorded even after resection of the renal capsule and also after injecting procaine into the pelvis. These indicate that the impulses recorded were not from the nerve endings of the capsule nor from pelvis. ${ }^{12}$ Histologically, the nerve endings which were considered to join the myelinated afferent fibers were observed in the adventitia of the spiral arteries and their surrounding connective tissues, connective tissues of the renal sinus, the surrounding tissues of the glomerular arteries, and also in the renal veins. ${ }^{2,4), 6)-8}$ It is most probable that the impulses examined in the present experiments were from the nerve endings in the interstitial tissues, because the impulses had close relations with the changes in the intrarenal pressure and because the major component of the pressure is the interstitial pressure. ${ }^{5), 13 \text { ) }}$

There remains the possibility, however, that the regularly discharging type A impulses were from the nerve endings in the small arteries like the spiral artery whose pressure is very low and who has a phasic delay from the extrarenal arterial pressure waves, ${ }^{13)}$ and that the nerve endings were excited compressed by the distended neighbouring veins when the venous pressure was altered to different direction from the intrarenal and renal arterial pressures.

It was not investigated whether these two types of the impulses were from the afferent myelinated fibers and whether there exists a functional difference between them. 


\section{RefERENCES}

1. Aars, H. and Akre, S.: Acta physiol. Scand. 78 : 184, 1970.

2. Abraham, V. C. : Acta Biol. Acad. Hung. 4 : 307, 1953.

3. Aström, A, and Crafoord, J. : Acta Physiol. Scand. 170: 10, 1967.

4. Fujiwara, S. : J. Jap. Soc. Nephrol. $10: 301,1968$.

5. Gottschalk, C. W.: Am. J. Physiol. 169 : 180, 1952.

6. Harman, P. J. and Davis, H. : J. Comp. Neurol. 89 : 225, 1948.

7. Knoche, H.: Z. Zellforsch. $36: 448$, 1951.

8. De Mylder, C. G. : Arch. de Biol. 52 : 509, 1941.

9. Nijima, A.: J. Jap. Soc. Physiol. $21: 477,1964$ (in Japanese).

10. Nijima, A.: J. Jap. Soc. Physiol. $27: 72,1965$ (in Japanese).

11. Pines, Y. L.: Fiziol. Zh. SSSR, Sechenov. 45: 1139, 1959.

12. Ueda, H., Uchida, Y., and Kamisaka, K. : Jap. Heart J. 8 : 597, 1967.

13. Yamada, S. and Åström, A. : Am. J. Physiol. 196: 213, 1959. 